\title{
Erratum to: The pattern of acute injuries in patients from alpine skiing accidents has changed during 2000-2011: analysis of clinical and radiological data at a level I trauma center
}

\author{
Marius C. Wick · Christian Dallapozza $\cdot$ Markus Lill \\ Cecilia Grundtman · Iris E. Chemelli-Steingruber • \\ Michael Rieger
}

Published online: 22 August 2013

(C) Springer-Verlag Berlin Heidelberg 2013

\section{Erratum to: Arch Orthop Trauma Surg}

DOI 10.1007/s00402-013-1822-6

The original article has been published with incorrect corresponding author name. The corresponding author should be Christian Dallapozza.

The online version of the original article can be found under doi:10.1007/s00402-013-1822-6.

M. C. Wick · I. E. Chemelli-Steingruber · M. Rieger

Department of Radiology, Innsbruck Medical University,

Anichstrasse 35, 6020 Innsbruck, Austria

e-mail: marius.wick@i-med.ac.at

I. E. Chemelli-Steingruber

e-mail: iris.chemelli-steingruber@i-med.ac.at

M. Rieger

e-mail: michael.rieger@tilak.at

C. Dallapozza $(\varangle) \cdot$ M. Lill

Department for Trauma Surgery and Sports Medicine, Innsbruck

Medical University, Innsbruck, Austria

e-mail: christian.dallapozza@uki.at

M. Lill

e-mail: markus.lill@auva.at

C. Grundtman

Laboratory of Autoimmunity, Division of Experimental

Pathophysiology and Immunology, Biocenter, Innsbruck Medical

University, Innsbruck, Austria

e-mail: cecilia.grundtman@i-med.ac.at 